\title{
Training program developed for senior undergraduates majoring in optical communication
}

Sheng Cui, Xinliang Zhang, Changjian Ke

Sheng Cui, Xinliang Zhang, Changjian Ke, "Training program developed for senior undergraduates majoring in optical communication," Proc. SPIE 10452, 14th Conference on Education and Training in Optics and Photonics: ETOP 2017, $104522 Z$ (16 August 2017); doi: 10.1117/12.2269687

SPIE Event: 14th Conference on Education and Training in Optics and Photonics, ETOP 2017, 2017, Hangzhou, China 


\title{
Training program developed for senior undergraduates majoring in optical communication
}

\author{
Sheng Cui*a , Xinliang Zhang ${ }^{\mathrm{a}}$, Changjian Ke ${ }^{\mathrm{a}}$ \\ ${ }^{a}$ School of optical and electronic information, Huazhong University of Science and Technology, \\ 1037 Luoyu Road, Wuhan, Hubei, China 430074
}

\begin{abstract}
Based on the well-known simulation software VPI TransmissionMaker, a comprehensive training program for senior undergraduates majoring in optical communication and optical network technology was developed by the author after detailed study of the teaching difficult and key points in the discipline. Aiming at solving practical scientific and engineering problems, the program helped our students to develop the ability of acquiring and applying knowledge by designing optical devices, optical signal processing algorithms and optical fiber communication systems. Furthermore, innovation is inspired by introducing competition mechanism among project teams. The program was validated through four years of use and achieved good results.
\end{abstract}

Keywords: Training program, senior undergraduates, optical communication, professional simulation software, problem-based learning

\section{INTRODUCTION}

Optical communication technology is developing at a faster pace than Moore's law, and the capacity of fiber optic communication systems will double every 9 months. With the acceleration of technological upgrading, the teaching contents and methods should be renovated. Passive receptive learning should be replaced by problem-based (PBL) active learning [1]. PBL aims at scientific or engineering problems at the beginning point of a certain course of study and has been widely adopted in teaching engineering in Europe and North America universities. It can help students arrive at a more profound understanding of fundamental knowledge and acquire more problem-solving skills.

This paper proposes an approach to implement PBL in teaching senior undergraduates optical fiber communication technology. It has been a part of the syllabus reform in School of Optical and Electronic Information, Huazhong University of Science and Technology since four years ago. Students are encouraged to acquire knowledge and skills by solving real scientific and engineering problems elaborately selected. These problems are chosen according to the teaching difficult and key points in this discipline so that this training program is well integrated with the overall professional courses. The solutions proposed by the students can be verified using the well-known professional optical communication system simulation software VPI TransmissionMaker [2]. This software is widely used in academics and companies as an efficient analysis and design tool. To our knowledge this attempt is the first of its kind in China.

\section{DESIGN PRINCIPLES OF THE TRAINING PROGRAM}

The four related compulsory courses for the senior undergraduates majoring in optical communication in our school are shown in Fig.1. This figure also shows the relationship between them. The course at a higher level is based on the one at the lower level. Three of them teach theories and methods. While the training program implemented as a course project focuses on the application of the theories, methods learned in the other three courses. The objectives of the problem based training program are to cultivate the follow skills:

- Analytical skill to analyze the trend of the development and find the main problem.

- Critical thinking skills to find the best solutions

- Mathematical modeling skills to use numerical simulation models to study and solve different problems.

*cuisheng@hust.edu.cn; phone 86 15902797167; fax 8627 87556188;

14th Conference on Education and Training in Optics and Photonics: ETOP 2017, edited by Xu Liu,

Xi-Cheng Zhang, Proc. of SPIE Vol. 10452, 104522Z · @ 2017 ICO, IEEE, OSA, SPIE

CCC code: $0277-786 X / 17 / \$ 18 \cdot$ doi: $10.1117 / 12.2269687$

Proc. of SPIE Vol. 10452 104522Z-1 
- Communication and written skills to give oral presentations and write research reports.

- Teamwork skills to lead a team or collaborate with other team members.

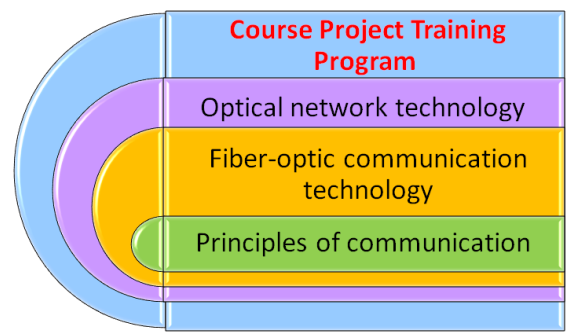

Figure 1. The professional courses and the relation between them.

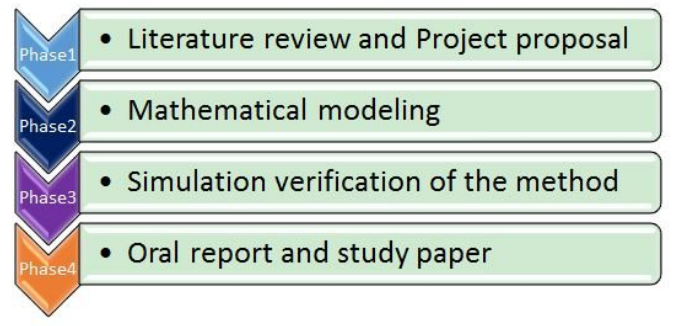

Figure 2. The implementation process of the training program.

To realize the above objectives the training program is carried out in four phases as shown in Fig.2. In the first phase the project team is required to find and analyze the most important literatures and cutting-edge product data sheets, so that they can learn how to correctly identify the requirements and main problem. In some cases the students are not perceptive enough and have sufficient background knowledge to identify the problem, so the facilitator should keep interacting with them and be a part of the feedback loop that helps them remain in the correct direction. Once the problem is confirmed, a thorough research is expected to find the possible solutions. The students are encouraged to compare the different solutions proposed before and point out the pros and cons of them. The facilitator will then guide them to find their own best solution by organizing a brain-storming and discussion session. In the end the project team is expected to deliver a literature review and project proposal describing the problems and solutions. In this phase the analytical and critical thinking skills are cultivated and some creative ideas may also be generated.

In the second phase, the students are required to develop a quantitative analysis method based on VPI TransmissonMaker to verify and improve their solutions. This software has a graphic user interface and simulation modules for devices and measuring apparatus commonly used in the field of optical communication. Novel or customized devices can also be created by co-simulation with Matlab or other languages. The ease of use can help the students to develop a simulation model more quickly. The model and parameters are checked by the facilitator to guarantee that the results are reliable.

In the third phase the students are expected to analyze the performance of their solutions according to the practical requirements identified in the first phase. In this phase the students can learn how to obtain and analyze technical data and how to draw instructive graph and table to demonstrate the test results. They can also find the limitations and shortages of their solutions. With the help of the facilitator they are expected to modify the original scheme and obtain better results.

In the fourth phase the students are required to give an oral presentation and write a research paper to elaborate their findings and solutions. They are evaluated by their performance in the presentation and the quality of their paper. The evaluation also put sufficient weighing on their performance through the whole process. With the above four phases the objectives of the problem based training program can be realized effectively. 


\section{EXAMPLES OF THE TRAINING PROGRAM}

As shown in Fig.3, the problems set for the students can be classified into three classes: 1) optical transmission systems, like the long-haul and access networks; 2) optoelectronic devices, like optical amplifiers, optical transceivers, all-optical optical wavelength converters and switches; 3) algorithms, like the chromatic and polarization model dispersion compensation algorithms used in digital coherent optical receivers.

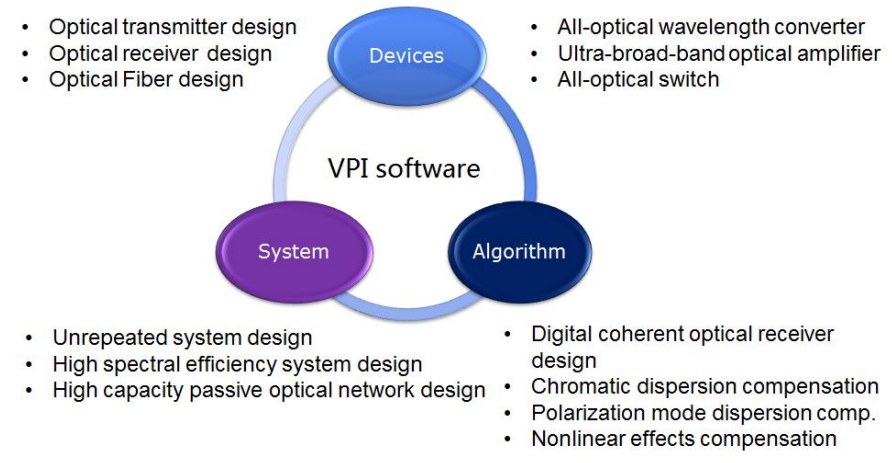

Figure 3.The classification and examples of the problems set for students.

For example, one problem is to design an unrepeated optical transmission system and find the underlying mechanisms limiting the transmission distance. This problem involves the consideration of fiber loss, chromatic dispersion and nonlinearity. To deal with these problems, students are required to probe deeper into the knowledges they have learned to find the appropriate techniques. For example by designing an ultra-low pure silica core loss large effective area optical fiber, the loss and nonlinearity can be reduced. By utilizing remotely pumped EDFA or distributed Raman amplifier the loss can be compensated. By using binary phase shift keying (BPSK) modulation and coherent detection the sensitivity can improved. By employing nonlinear compensation techniques such as digital backward propagation, mid-link optical phase conjugation (OPC) the nonlinear distortions can be mitigated. By solving the problem, the knowledges they learn in different courses are no more isolate but appear in relationship and the knowledges are integrated in system approach.

\section{CONCLUSIONS}

In this paper we present a problem-based training program utilizing professional VPI software for senior undergraduates majoring in optical communication. The training objectives, implementation process and setting of the problems are introduced. From the four years of practice, the approach is effective in developing the important skills expected for a researcher or engineer in the field. More elaborately selected problems can be developed with the same approach to keep up with the newest development in the field.

\section{REFERENCE}

[1] Lucas, Bill, and Janet Hanson., "Thinking like an engineer: Using engineering habits of mind and signature pedagogies to redesign engineering education," International Journal of Engineering Pedagogy 6(2), 4-13 (2016).

[2] VPI Photonics, “Description of VPItransmissionMaker,” http://www.vpiphotonics.com/Tools/OpticalSystems 